\title{
Spin Phase Transition in a Correlated Composite Fermion Liquid
}

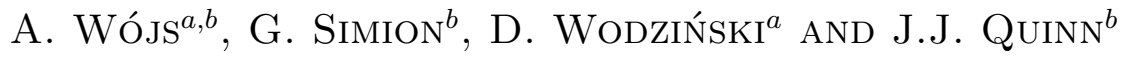 \\ ${ }^{a}$ Institute of Physics, Wrocław University of Technology \\ Wybrzeże Wyspiańskiego 27, 50-370 Wrocław, Poland \\ ${ }^{b}$ Department of Physics, University of Tennessee, Knoxville, TN 37996, USA \\ We study spin polarization of the $\nu_{\mathrm{e}}=4 / 11$ fractional quantum Hall \\ state corresponding to the $\nu=1 / 3$ filling of the second composite fermion \\ Landau level, and predict a spin phase transition in realistic systems.
}

PACS numbers: 71.10.Pm, 73.43.-f

\section{Introduction}

The composite fermion $(\mathrm{CF})$ model [1] has been very successful in the explanation of incompressibility of a whole family of fractional quantum Hall $(\mathrm{FQH})$ states [2] at the "Jain series" of the Landau level (LL) filling factors $\nu_{\mathrm{e}}=n /(2 n p \pm 1)$. At these fillings, the CF transformation involving attachment of an even number $2 p$ of magnetic flux quanta $\phi_{0}=h c / e$ to each electron converts a partially filled LL of electrons with strong (Coulomb) interaction into a small number $\nu_{\mathrm{CF}} \equiv n$ of completely filled LL's of nearly noninteracting CF's (the general relation between electron and CF filling factors being $\nu_{\mathrm{CF}}^{-1}=\nu_{\mathrm{e}}^{-1}-2 p$ ). In filled shells, the weak residual $\mathrm{CF}-\mathrm{CF}$ interactions play no role and the incompressibility of $\nu_{\mathrm{e}}=n /(2 n p \pm 1)$ states is said to result from a single-particle cyclotron gap of the CF's in the reduced magnetic field $B^{*}=B-2 p \phi_{0} \varrho$, where $\varrho$ is the $2 \mathrm{D}$ electron/CF concentration (connected to $\nu_{\mathrm{e}}=2 \pi \varrho \lambda^{2}$ via magnetic length $\lambda=\sqrt{\hbar c / e B})$.

Recently, Pan et al. [3] have observed the FQH effect in a spin-polarized electron gas at a series of filling factors between two neighboring Jain states, i.e. at $1 / 3<\nu_{\mathrm{e}}<2 / 5$, and thus corresponding to the fractional $\mathrm{CF}$ filling factors, $1<\nu_{\mathrm{CF}}<2$. In particular, the incompressible states observed at $\nu_{\mathrm{e}}=4 / 11,3 / 8$, $5 / 13$ correspond to $\nu_{\mathrm{CF}}=4 / 3,3 / 2$, and $5 / 3$, respectively. Clearly, incompressibility of these states must depend on the $\mathrm{CF}-\mathrm{CF}$ interaction within a partially filled second CF LL. 
The familiar values of second-CF-LL fillings, $\nu \equiv \nu_{\mathrm{CF}}-1=1 / 3,1 / 2$, and $2 / 3$, respectively, suggested analogy [4] to electron states at the same fillings of the same (second) electron LL, i.e. to the FQH effect at $\nu_{\mathrm{e}}=2+\nu$ ("2" due to spin degeneracy of the first electron LL), i.e. at $\nu_{\mathrm{e}}=7 / 3,5 / 2$, and $8 / 3$. However, the analogy fails [5] due to the qualitatively different short-range e-e and $\mathrm{CF}-\mathrm{CF}$ interaction pseudopotentials (interaction energy $V$ as a function of pair angular momentum $\mathcal{R}$ ) in their respective second LL's. In fact, despite active research [6-8], the nature of $\nu_{\mathrm{e}}=4 / 11,3 / 8$, and $5 / 13$ states is not yet completely understood.

In this paper we study spin polarization of the $\nu_{\mathrm{e}}=4 / 11$ state, motivated by the troubling fact that the spin-polarized state has been observed [3] but it is not well understood, while the unpolarized state which appears to be much easier to understand (analogy to the electron Laughlin state works) has not yet been reported in experiment. The main result is the spin phase diagram, from which we predict a spin transition at $\nu_{\mathrm{e}}=4 / 11$, induced, e.g. by an additional electric field narrowing the electron layer.

\section{Results and discussion}

The numerical (exact-diagonalization) calculations were carried out in the Haldane geometry [9]. In this model, $N$ particles (e.g., CF's) are confined to a sphere, with the normal magnetic field $B$ yielding the desired LL degeneracy $g=2 Q+1$ produced by a Dirac monopole of strength $2 Q \phi_{0}$ in the center. To avoid finite-size errors, the calculations are repeated for different $(N, g)$ and the results are extrapolated to $N \rightarrow \infty$ and $N / g \rightarrow \nu$.

To compare the energies of polarized and unpolarized states at $\nu_{\mathrm{e}}=4 / 11$ we must calculate and compare the single-particle energies $\varepsilon$ of the appropriate quasiparticles (QP's) and their many-body correlation energies $u$. For the polarized state these QP's are the Laughlin quasielectrons (QE's) [10] in the second spin- $\uparrow$ CF LL. For the unpolarized state the QP's are the reversed-spin Rezayi [11] $\mathrm{QE}_{\mathrm{R}}$ 's in the lowest spin- $\downarrow$ CF LL. In the CF picture, $\Delta \varepsilon=\varepsilon_{\mathrm{QE}}-\varepsilon_{\mathrm{QER}}$ is the difference between the $\mathrm{CF}$ cyclotron and the Zeeman energies.

The actual values of $\varepsilon$ can be extracted $[12,13]$ from an exact diagonalization of finite electron systems. In Fig. 1a they are drawn as a function of width $w$ of the quasi-2D electron layer, with the electron wave function in the normal direction taken as $\chi(z) \propto \cos (z \pi / w)$. To put the shown width range in perspective, let us note that a $12 \mathrm{~nm}$ well at $B=10 \mathrm{~T}$ corresponds to $w / \lambda=1.9$, and a $40 \mathrm{~nm}$ well at $B=23 \mathrm{~T}$ corresponds to $w / \lambda=8.1$.

The short-range $\mathrm{QE}-\mathrm{QE}$ and $\mathrm{QE}_{\mathrm{R}}-\mathrm{QE}_{\mathrm{R}}$ pseudopotentials $V(\mathcal{R})$ are obtained from the exact diagonalization [5] or Monte Carlo [14] calculations of a finite number of electrons. The long-range behavior of $V(\mathcal{R})$ results from the known $-e / 3$ electric charge of $\mathrm{QE}$ 's and $\mathrm{QE}_{\mathrm{R}}$ 's. Matching the two limits, we obtain the result shown in Fig. $1 \mathrm{~b}$ for $w=0$. 


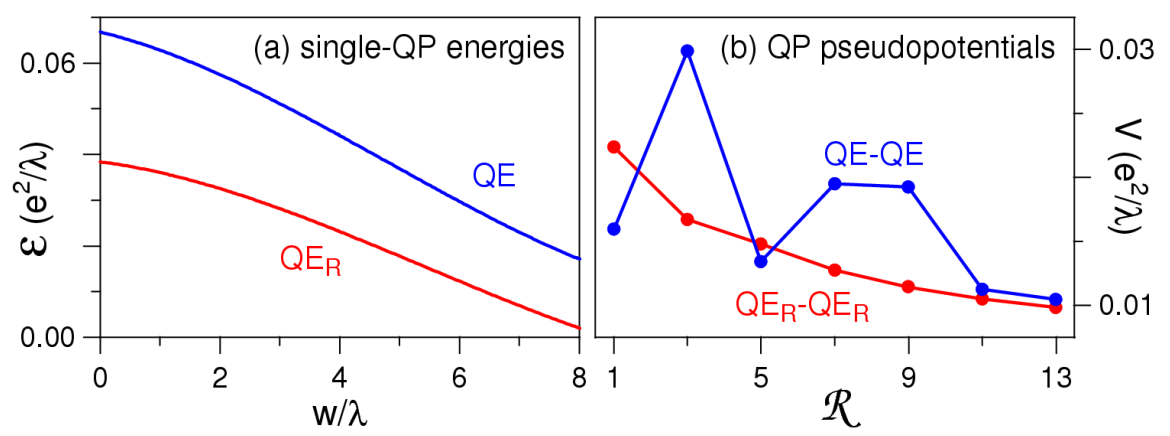

Fig. 1. (a) Dependence of the $\mathrm{QE}$ and $\mathrm{QE}_{\mathrm{R}}$ energies $\varepsilon$ on electron layer width $w$. (b) QE's and $\mathrm{QE}_{\mathrm{R}}$ 's interaction pseudopotentials $V(\mathcal{R})$ in an ideal 2D layer $(w=0)$.
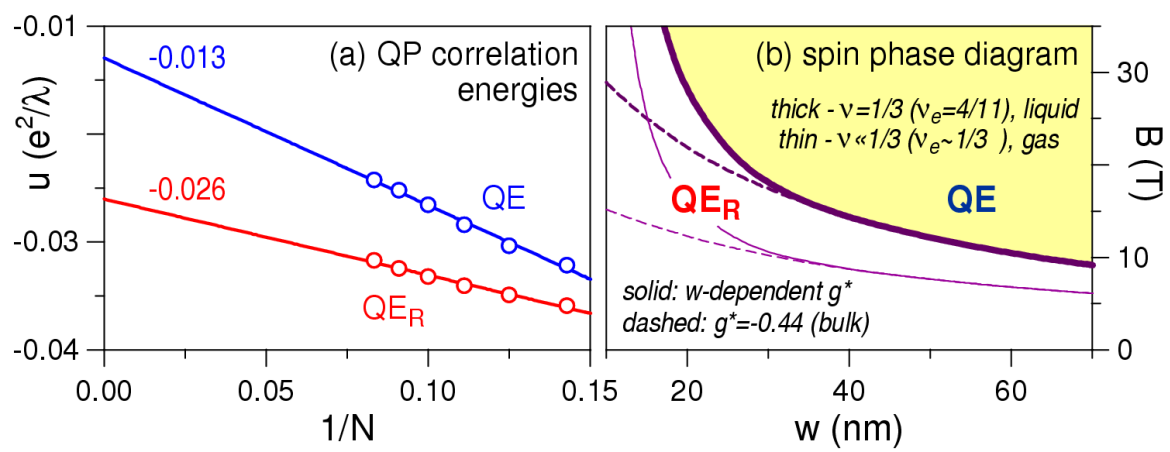

Fig. 2. (a) Correlation energy $u$ in the $\nu=1 / 3$ state of QE's or QE $E_{\mathrm{R}}$ 's as a function of their inverse number $N^{-1}$ in an ideal $2 \mathrm{D}$ layer $(w=0)$. (b) Phase diagram (critical layer width $w$ vs. magnetic field $B$ ) for the $\mathrm{QE}-\mathrm{QE}_{\mathrm{R}}$ spin transition at $\nu_{\mathrm{e}}=4 / 11$, assuming the effective (width dependent) electron Landé $g^{*}$-factor for GaAs. For comparison, the thick dashed line is for a constant (bulk) value $g^{*}=-0.44$. Two thin lines additionally ignore the correlation energy $u$ (adequately for $\nu \ll 1$ ).

The many-QP correlation energy per particle is defined as $u=(E+$ $\left.U_{\text {bckg }}\right) / N$, where $E$ is the interaction energy of the ground state of $N$ QP's and $U_{\mathrm{bckg}}=-(N q)^{2} / 2 R$ is a correction due to the interaction with the charge-compensating background (with the sphere radius $R=\lambda \sqrt{Q}$ ). In Fig. 2a we plot $u(N)$ obtained for $w=0$ from diagonalization of $N \leq 12$ QP's. The results of extrapolation are $u_{\mathrm{QER}}=-0.026 e^{2} / \lambda$ (very close to the value for an electron Laughlin state when the charge difference $e \rightarrow e / 3$ is taken into account) and $u_{\mathrm{QE}}=-0.013 e^{2} / \lambda$. The difference $\Delta u=u_{\mathrm{QE}}-u_{\mathrm{QER}}$ was recalculated in a similar manner for various widths $w$. Whether $\mathrm{QE}$ 's or $\mathrm{QE}_{\mathrm{R}}$ 's will form a $\nu=1 / 3$ state at $\nu_{\mathrm{e}}=4 / 11$ depends on the competition of the Coulomb and Zeeman energies. The condition for the $\mathrm{QE} \leftrightarrow \mathrm{QE}_{\mathrm{R}}$ transition is $\Delta \varepsilon+\Delta u=E_{\mathrm{Z}}$. In Fig. 2b we drew the spin phase diagram assuming width dependence on the effective Landé factor 
$g^{*}$ appropriate for GaAs wells [15]. Clearly, the spin transition in narrower wells shifts quickly to higher $B$ (i.e., to higher $\varrho_{\mathrm{e}} \propto B$ ). The role of QP-QP interaction in stabilizing the $\mathrm{QE}_{\mathrm{R}}$ phase is evident from the comparison of boundaries dividing correlated $\mathrm{QE} / \mathrm{QE}_{\mathrm{R}}$ liquids (thick lines) and non-interacting $\mathrm{QE} / \mathrm{QE} \mathrm{R}_{\mathrm{R}}$ gases (thin lines).

\section{Acknowledgments}

The authors thank Wei Pan for helpful discussions and sharing unpublished experimental data, and acknowledge partial support from grants DE-FG 02-97ER45657 of US DOE and N202-071-32/1513 of the Polish Ministry of Science and Higher Education.

\section{References}

[1] J.K. Jain, Phys. Rev. Lett. 63, 199 (1989); Science 266, 1199 (1994).

[2] D.C. Tsui, H.L. Störmer, A.C. Gossard, Phys. Rev. Lett. 48, 1559 (1982).

[3] W. Pan, H.L. Störmer, D.C. Tsui, L.N. Pfeiffer, K.W. Baldwin, K.W. West, Phys. Rev. Lett. 90, 016801 (2003).

[4] J.H. Smet, Nature (London) 422, 391 (2003).

[5] A. Wójs, J.J. Quinn, Phys. Rev. B 61, 2846 (2000).

[6] C.C. Chang, S.S. Mandal, J.K. Jain, Phys. Rev. B 67, 121305(R) (2003).

[7] A. Wójs, K.S. Yi, J.J. Quinn, Phys. Rev. B 69, 205322 (2004); A. Wójs, D. Wodziński, J.J. Quinn, ibid. 71, 245331 (2005); ibid. 74, 035315 (2006).

[8] M.O. Goerbig, P. Lederer, C.M. Smith, Phys. Rev. B 69, 155324 (2004).

[9] F.D.M. Haldane, Phys. Rev. Lett. 51, 605 (1983).

[10] R.B. Laughlin, Phys. Rev. Lett. 50, 1395 (1983).

[11] E.H. Rezayi, Phys. Rev. B 36, 5454 (1987); ibid. 43, 5944 (1991).

[12] G. Fano, F. Ortolani, E. Colombo, Phys. Rev. B 34, 2670 (1986).

[13] I. Szlufarska, A. Wójs, J.J. Quinn, Phys. Rev. B 64, 165318 (2001).

[14] S.Y. Lee, V.W. Scarola, J.K. Jain, Phys. Rev. Lett. 87, 256803 (2001).

[15] M.J. Snelling, G.P. Flinn, A.S. Plaut, R.T. Harley, A.C. Tropper, R. Eccleston, C.C. Phillips, Phys. Rev. B 44, 11345 (1991). 